\title{
A new insight into in vitro behaviour of poly( $\varepsilon$-caprolactone)/bioactive glass composites in biologically related fluids
}

\author{
Michal Dziadek $^{1, \star}$ (D), Barbara Zagrajczuk ${ }^{1}$ (D), Elzbieta Menaszek $^{2}$ (D), and Katarzyna Cholewa-Kowalska ${ }^{1}$ (1) \\ ${ }^{1}$ Department of Glass Technology and Amorphous Coatings, Faculty of Materials Science and Ceramics, AGH University of Science \\ and Technology, 30 Mickiewicza Ave., 30-059 Kraków, Poland \\ ${ }^{2}$ Department of Cytobiology, Collegium Medicum, Jagiellonian University, 9 Medyczna St., 30-688 Kraków, Poland
}

Received: 25 September 2017

Accepted: 18 November 2017

Published online:

27 November 2017

(C) The Author(s) 2017. This article is an open access publication

\begin{abstract}
In the present work, the role of content, size and chemical composition of gel-derived bioactive glass particles from the $\mathrm{SiO}_{2}-\mathrm{CaO}-\mathrm{P}_{2} \mathrm{O}_{5}$ system in modulating the in vitro bioactivity, osteoinductive properties and long-term (up to 15 months) degradation behaviour of poly( $\varepsilon$-caprolactone)-based composite films was investigated. Bioactivity was assessed in simulated body fluid (SBF) and HEPES-free Dulbecco's modified Eagle medium (DMEM) supplemented with 10\% foetal bovine serum (FBS), while hydrolytic degradation tests were performed in phosphate buffer saline. Obtained composite films showed excellent calcium phosphate (CaP) layer forming ability in both SBF and DMEM-10\% FBS. However, kinetics of bioactivity process strongly depended on the type of medium used. The layer of amino acids and proteins, derived from cell culture medium, on the surfaces of composites created barrier that inhibited release of the ions on the one hand, while increasing nucleation density of calcium phosphates, affecting the morphology of formed CaP layers on the other. The presence of bioactive glass fillers was shown to impart osteoinductive properties to obtained films, supporting osteoblast attachment and proliferation, as well as stimulating cell differentiation and also matrix mineralization process in vitro. We showed that kinetics of bioactivity process and also osteoinductive properties of composite films could be easily modulated with the use of different contents and chemical compositions of fillers. The results showed that modification of PCL matrix with bioactive glass particles accelerated its degradation. We proved that the degradation rate of composites could be controlled and optimized for bone regeneration, in particular by using bioactive fillers causing different calcium phosphate layer forming ability on the surfaces of composites, depending on particle size and chemical composition. We have presented new opportunities to design and obtain multifunctional composites with tunable degradation and bioactivity kinetics, as well as biological properties that can meet complex requirements of bone tissue engineering.
\end{abstract}

Address correspondence to E-mail: dziadek@agh.edu.pl 


\section{Introduction}

Biomaterials for bone tissue engineering (BTE) applications should degrade progressively at a rate matching the regeneration of new bone in vivo to allow full restoration of native tissue structure and functions $[1,2]$. The degradation behaviour of the materials should vary based on target applications, namely material form (e.g. scaffold, barrier membrane) and implantation site. The gradual resorption of three-dimensional porous scaffolds, used for filling bone defects, allows to create space for the new bone tissue growth, while the necessary structural support is provided until full regeneration of the tissue [2]. Porous membranes are mainly used for guided bone regeneration (GBR), as a physical barrier to prevent connective tissue ingrowth into the bone defects and to maintain a suitable space for bone regeneration processes. The controlled degradation rate of the membrane allows to maintain its mechanical integrity and thus spacemaintaining properties over the entire period of bone regeneration on the one hand and to exclude the need for its surgical removal on the other [3, 4]. Furthermore, an increasing trend towards the use of polymer-based scaffolds and membranes as carriers for local drug delivery has been observed in recent years. In that case, polymer degradation rate is the key factor affecting drug release kinetics [5]. Therefore, manufacturing materials with controllable degradation behaviour is essential for bone repair and regeneration and still represents a big challenge in modern BTE.

Relatively high mechanical strength and high cytocompatibility with osteoblasts make poly( $\varepsilon$-caprolactone) (PCL) suitable for bone tissue regeneration $[6,7]$. However, because of a high degree of crystallinity and hydrophobicity, PCL shows low degradation rate (up to 4 years, depending on the initial molecular weight, crystallinity, implant form, porosity, etc.) [8], which limit its use in some BTE applications. Degradation rate of PCL can be modified by its copolymerization or blending with others, faster degrading aliphatic polyesters [e.g. poly(L-lactide), poly(DL-lactide)] $[1,5,9]$. Another way of accelerating PCL degradation is modification with hydrophilic ceramic fillers (e.g. nanoHAp, TCP, bioactive glass and calcium silicate particles) $[8,10,11]$ that affect surface and bulk properties of the material, such as wettability, water absorption and polymer matrix crystallinity. However, there is limited number of research concerning the influence of the chemical composition of ceramic fillers on degradation behaviour of aliphatic polyesters.

The direct bone bonding ability through the formation of a bone-like hydroxyapatite (HAp) phase on the material surface without an intermediate layer of modified tissue is the main advantage of the so-called bioactive glasses [12]. Moreover, reactions on the material surface could induce the release of critical concentrations of soluble $\mathrm{Si}, \mathrm{Ca}, \mathrm{P}$ and $\mathrm{Na}$ ions, which can lead to favourable intracellular and extracellular responses, actively stimulating bone formation [13]. Such bioactive glasses belong to the group of osteoinductive biomaterials. In the group of silicate bioactive glasses, those obtained using sol-gel method exhibit much higher surface area and the presence of siloxane groups $(\mathrm{Si}-\mathrm{OH})$ in their structure. Thus, their bioactivity and cellular response are usually enhanced compared to conventional meltderived glasses [14]. Therefore, the combination of PCL matrix with gel-derived bioactive glass fillers creates possibility of not only modulating material degradation rate but also imparting osteoinductive and bioactive properties.

The evaluation of the biomaterial bioactivity usually refers to the identification of the HAp layer on its surface in a living organism (in vivo) or in simulated body environment (in vitro) [12]. The so-called simulated body fluid (SBF) was developed by Kokubo and has been the most widely used solution for in vitro bioactivity tests recently [15]. However, SBF cannot simulate physiological conditions in a living body completely, because it only substitutes inorganic part of blood serum. The three main differences between SBF solution and blood serum can be listed as follows: (1) the absence of organic species, such as proteins, (2) the presence of TRIS (tris-[(hydroxymethyl)aminomethane]) to buffer SBF solution and (3) the absence of carbonate content control in SBF solution, although carbonates act as $\mathrm{pH}$ buffer in serum [16]. Therefore, in the literature, attempts to substitute SBF with the commercially available cell culture media, i.e. HEPES-free Dulbecco's modified Eagle medium (DMEM) solution, have been reported $[15,17]$. Apart from the inorganic part of blood serum, this solution contains also the organic component (vitamins, amino acids, etc.). Moreover, DMEM can be supplemented with foetal bovine serum (FBS), containing additional organic species, such as proteins, to obtain complete growth medium for certain cell lines. The medium can be carbonate 
buffered as the test is performed in a $37{ }^{\circ} \mathrm{C}$ incubator under a humidified atmosphere of $95 \%$ air and 5\% $\mathrm{CO}_{2}$, which better simulates the in vivo environment $[15,17]$.

In the present work, the effect of content, size and chemical composition of gel-derived bioactive glass particles from the $\mathrm{SiO}_{2}-\mathrm{CaO}-\mathrm{P}_{2} \mathrm{O}_{5}$ system on the in vitro bioactivity, osteoinductive properties and long-term degradation behaviour of PCL-based composite films was investigated. Calcium phosphate layer forming ability was assessed in SBF and DMEM supplemented with 10\% FBS. Degradation kinetics of composites was examined by incubation them in phosphate buffer saline (PBS) up to 15 months.

\section{Materials and methods}

\section{Materials}

Poly( $\varepsilon$-caprolactone) (PCL; $\mathrm{Mn} 80 \mathrm{kDa}, \mathrm{Mw} /$ $\mathrm{Mn}<2$; Sigma-Aldrich, USA) and two bioactive glasses (SBGs) of different compositions (S2 and A2) as composite components were used. Bioactive glasses of the following compositions (mol\%) S2: $80 \mathrm{SiO}_{2}-16 \mathrm{CaO}-4 \mathrm{P}_{2} \mathrm{O}_{5}$ and $\mathrm{A} 2: \quad 40 \mathrm{SiO}_{2}-54 \mathrm{CaO}-$ $6 \mathrm{P}_{2} \mathrm{O}_{5}$ were produced using the sol-gel method described in our previous work [18]. In order to prepare films, dichloromethane (DCM, POCh, Poland) was used.

\section{Bioactive glass preparation}

Tetraethoxysilane (TEOS; $\left.\mathrm{Si}\left(\mathrm{OC}_{2} \mathrm{H}_{5}\right)_{4}\right)$, triethylphosphate (TEP; $\left.\mathrm{OP}\left(\mathrm{OC}_{2} \mathrm{H}_{5}\right)_{3}\right)$ (Sigma-Aldrich, USA) and calcium nitrate tetrahydrate $\left(\mathrm{Ca}\left(\mathrm{NO}_{3}\right)_{2} \cdot 4 \mathrm{H}_{2} \mathrm{O}\right)(\mathrm{POCh}$, Poland) were used as basic components of the sol-gel process. $1 \mathrm{M} \mathrm{HCl}$ solution (POCh, Poland) was used as a catalyst in the hydrolysis and condensation reactions. The molar ratio of TEOS:TEP:Ca $\left(\mathrm{NO}_{3}\right)_{2}$ :$\mathrm{H}_{2} \mathrm{O}: \mathrm{HCl}$ was 1:0.3:1.4:4:0.2 for A2 glass and 1:0.15:0.25:3:0.2 for S2 glass, respectively. The formed gel was gradually dried at temperature, increasing in the range from 40 to $120{ }^{\circ} \mathrm{C}$ for 7 days and then subjected to thermal treatment at $700{ }^{\circ} \mathrm{C}$ for $20 \mathrm{~h}$. Two glass particle sizes were obtained: $<45 \mu \mathrm{m}$ by grinding and sieving and $<3 \mu \mathrm{m}$ by milling in an attritor with $\mathrm{ZrO}_{2}$ balls in isopropyl alcohol medium. Particle size distributions in powder aqueous suspensions were analysed using laser diffraction method (Mastersizer 2000, Malvern, UK).

\section{Composite film preparation}

The PCL-bioactive glass composites were fabricated according to our previous work [19]. The glass particles were mixed with $5 \mathrm{w} / \mathrm{v} \%$ PCL solutions in DCM, on a magnetic stirrer for $24 \mathrm{~h}$. Afterwards, the suspensions were cast onto glass Petri dishes (diameter $90 \mathrm{~mm}$ ), which were covered with glass Petri dish lids (diameter $100 \mathrm{~mm}$ ) and placed in a clean place at room temperature for a slow evaporation. Subsequently, the films were dried under vacuum at ambient temperature to a constant weight. All films were stored at $4{ }^{\circ} \mathrm{C}$ in a desiccator until further investigation. The volume fraction of bioactive glass particles in composites was 12 and $21 \mathrm{vol} \%$. The reference films (pure PCL) were prepared by casting of PCL solutions in the same way as mentioned above. The surface that had been in contact with glass Petri dish during casting was marked as GS, while the other one was marked as AS.

\section{Material evaluation}

\section{In vitro bioactivity test}

In vitro bioactivity was evaluated by incubation of materials in simulated body fluid (SBF) prepared according to Kokubo [20] and in HEPES-free Dulbecco's modified Eagle medium (DMEM, SigmaAldrich, USA) supplemented with $10 \%$ foetal bovine serum (FBS, HyClone, USA). The samples were immersed in SBF and DMEM-10\% FBS and incubated for $1,3,7$ and 14 days. Incubation in SBF was conducted at $37^{\circ} \mathrm{C}$ in tightly closed, separate polypropylene containers in the incubator. The materials incubated in DMEM-10\% FBS were placed in separate polypropylene containers with gas permeable adhesive seals at $37{ }^{\circ} \mathrm{C}$ in $\mathrm{CO}_{2}$ incubator. The sample weight-to-SBF and DMEM-10\% FBS volume ratio was $10^{-3} \mathrm{~g} \mathrm{ml}^{-1}$. Afterwards, the samples were washed with anhydrous ethanol and air-dried at room temperature to a constant weight. GS surface of each sample was examined with SEM/EDX (Nova NanoSEM 200 FEI Europe Company) and ATR-FTIR (Bruker VERTEX $70 \mathrm{~V}$ spectrometer, USA) methods. The samples were covered with a carbon layer before SEM analysis. The ATR-FTIR spectra were registered 
with the use of a platinum single-crystal diamond ATR unit in the $550-4000 \mathrm{~cm}^{-1}$ wavenumber range, and 128 scans were accumulated at $4 \mathrm{~cm}^{-1}$ resolution. Furthermore, the changes in the concentration of calcium, phosphorus and silicon in the SBF and DMEM-10\% FBS during film incubation were analysed for each testing time using inductively coupled plasma atomic emission spectrometry (ICP-OES; Plasm 40, PerkinElmer, USA). The test was performed in triplicate and expressed as mean \pm standard deviation (SD).

\section{In vitro degradation study}

For degradation study, the films in the form of strips $(30 \times 5 \mathrm{~mm}, n=12)$ were placed in tightly closed polypropylene containers and incubated in phosphate buffer saline (PBS, $\mathrm{pH}=7.4$ ) at $37^{\circ} \mathrm{C}$ for 15 months. The PBS was exchanged once every month. The sample weight-to-PBS volume ratio was $10^{-3} \mathrm{~g} \mathrm{ml}^{-1}$. Degradation process was monitored by measuring $\mathrm{pH}$ before $\mathrm{PBS}$ changing ( $\mathrm{pH}$ meter, $\mathrm{CP}$ 315 Elmetron, Poland). Furthermore, weight changes, surface morphology, chemical composition, degree of crystallinity, melting temperature and tensile properties of the samples were examined after 1, 3, 6, 9, 12 and 15 months of incubation. The percentage of weight loss was calculated using the following equation: $\% W=\left(W_{\mathrm{i}}-W_{\mathrm{t}}\right) / W_{\mathrm{i}} \cdot 100 \%$, where $W_{\mathrm{i}}$ is the initial weight of the sample and $W_{t}$ is the weight of the dried sample after given time of the degradation test. Surface morphology and chemical composition were determined using scanning electron microscopy (SEM, Nova NanoSEM 200 FEI Europe Company) coupled with energy dispersion X-ray (EDX) analyser. The samples were covered with a carbon layer. The degree of crystallinity $\left(\chi_{c}\right)$ and melting temperature $\left(T_{\mathrm{m}}\right)$ of PCL were measured with power compensation differential scanning calorimetry (DSC, PerkinElmer DSC-7, USA). The melting temperature was determined at the maximum of the melting endotherm during a single heating run. The degree of crystallinity was estimated using the enthalpy of melting change according to the equation: $\chi_{c}=\Delta H_{\mathrm{m}} /(1-x) \Delta H_{\mathrm{m}}^{0}$, where $\Delta \mathrm{H}_{\mathrm{m}}$ and $\Delta H_{\mathrm{m}}^{0}$ were the enthalpies of melting of the sample and fully crystalline PCL (139.5 $\mathrm{J} \mathrm{g}^{-1}$ ) [8], respectively, and $x$ was the weight fraction of the bioactive glass particles. All of the samples (average weight $10 \mathrm{mg}$ ) were placed in standard aluminium pans. The specimens were scanned from 20 to $100{ }^{\circ} \mathrm{C}$ with the heating rate of $10^{\circ} \mathrm{C} \mathrm{min}{ }^{-1}$, using nitrogen as a purge gas. The results were averaged from the three measurements and were expressed as the mean \pm standard deviation (SD). Tensile strength $\left(\sigma_{\mathrm{M}}\right)$ and Young's modulus $\left(E_{\mathrm{t}}\right)$ were determined using a universal testing machine Inspect Table Blue $5 \mathrm{kN}$ with $100 \mathrm{~N}$ load cell (Hegewald \& Peschke, Germany). The preload force was $0.1 \mathrm{~N}$, and the test speed was $10 \mathrm{~mm} \mathrm{~min}{ }^{-1}$. Mechanical parameters were calculated by averaging ten measurements and were expressed as mean \pm standard deviation (SD).

\section{In vitro osteoblast response}

Material sterilization For the cell culture, films (round samples matching the size of wells of 48-well culture plate) were sterilized by soaking in $70 \%$ ethanol for $30 \mathrm{~min}$; further, both sides of samples were sterilized with UV-C light for $30 \mathrm{~min}$ each and washed with sterile phosphate buffered saline (PBS, HyClone, USA).

Cell culture The normal human osteoblasts (NHOst, Lonza, USA) were expanded in $75 \mathrm{~cm}^{2}$ tissue culture flasks (Nunc ${ }^{\mathrm{TM}}$, Denmark) in complete osteoblast growth medium OGM BulletKit (Lonza, USA) containing $10 \%$ FBS, $0.1 \%$ ascorbic acid and $0.1 \%$ GA1000 (gentamicin sulphate and amphotericin-B) at $37{ }^{\circ} \mathrm{C}$ in a humidified, $5 \% \mathrm{CO}_{2}$ atmosphere. The medium was changed every 3 days until a $70 \%$ confluent cell monolayer has developed. Then cells were detached from culture flasks using 5\% TrypsinEDTA (HyClone, USA), centrifuged and resuspended in fresh growth medium.

The prepared sterile films were placed at the bottom of 48-well culture plate wells (Nunc ${ }^{\mathrm{TM}}$, Denmark) and held by ultrapure silica glass inserts to prevent samples floating. NHOst cells were seeded on the GS surface of films at a density of $1.5 \cdot 10^{4}$ cells / $\mathrm{mL} /$ well and cultured for 3, 7, 14, 21 and 28 days in complete osteoblast growth medium OGM supplemented with differentiation kit SingleQuots (Lonza, USA), containing hydrocortisone-21-hemisuccinate and $\beta$-glycerophosphate. The bottom surfaces of tissue culture polystyrene (TCPS) wells served as a control.

Microscopic observation After 28 days of culture, films were rinsed with PBS, and then, cells were fixed 
with 3\% glutaraldehyde solution in sodium cacodylate buffer, $\mathrm{pH} 7.4$ (POCh, Poland) for $1 \mathrm{~h}$. Subsequently, the cells were dehydrated in graded series of ethanol solution (70, 80, 90, 96 and 100\%) and dried in air. Cell morphologies were evaluated using SEM (Nova NanoSEM 200 FEI Europe Company) after coating with carbon.

After 7 days of culture, the cells were stained with $0.01 \%$ acridine orange $(\mathrm{AO})$ solution (Sigma-Aldrich, USA) for $1 \mathrm{~min}$. Next, the samples were rinsed with PBS, observed and photographed under the fluorescence microscope (Olympus CX41, Japan).

Cell proliferation and material cytotoxicity In order to determine proliferation rate of NHOst cells and cytotoxic effect of obtained materials, ToxiLight ${ }^{\mathrm{TM}}$ BioAssay Kit and ToxiLight ${ }^{\mathrm{TM}} 100 \%$ Lysis Reagent Set (Lonza, USA) were used according to manufacturer's protocol. The kit was used to quantify adenylate kinase (AK) in both supernatant (representing damaged cells) and lysate (representing intact adherent cells). The results were expressed as mean \pm standard deviation (SD) from 8 samples for each experimental group.

Alkaline phosphatase activity Alkaline phosphatase (ALP) activity measurement is based on the hydrolysis reaction of 4-MUP (4-methylumbelliferyl phosphate, the substrate for ALP expressed by differentiated osteoblasts), to highly fluorescent product 4-MU (4-methylumbelliferone). After 7 and 14 days of culture, NHOst cells were disrupted via a cyclic freezing/thawing in order to release intracellular ALP. Cell lysates in triplicates were transferred to OptiPlate-96 microplate (PerkinElmer) and incubated with equal volumes of 4-MUP Liquid Substrate System (Sigma-Aldrich, USA) solution for $1 \mathrm{~h}$. Fluorescence was determined at 360/440 nm (excitation/ emission wavelengths) using POLARstar Omega microplate reader (BMG Labtech, Germany). The results were expressed as mean \pm standard deviation (SD) from 8 samples for each experimental group.

ECM mineralization Extracellular matrix (ECM) mineralization in cell culture was assessed using OsteoImage $^{\mathrm{TM}}$ Mineralization Assay (Lonza, USA). Cells were cultured with studied biomaterials for 14, 21 and 28 days; next, the samples were fixed, washed and stained according to the manufacturer's protocol. The assay is based on the specific binding of the fluorescent reagent to the hydroxyapatite portion of nodules deposited by cells. Fluorescence was read at 490/520 nm (excitation/emission wavelengths) using POLARstar Omega microplate reader. The results were expressed as mean \pm standard deviation (SD) from 4 samples for each experimental group.

\section{Statistical analysis}

The results were analysed using one-way analysis of variance (ANOVA) with Duncan post hoc tests, which were performed with Statistica 10 (StatSoft $^{\circledR}$, USA) software. The results were considered statistically significant when $p<0.05$.

\section{Results}

\section{In vitro bioactivity}

A2-PCL and S2-PCL composite films containing 12 and $21 \mathrm{vol} \%$ glass particles of $<45 \mu \mathrm{m}$ size and pure PCL material were examined in terms of in vitro bioactivity by soaking them in SBF and cell culture medium (DMEM supplemented with 10\% FBS) for 1, 3, 7 and 14 days. Figure 1 shows SEM images and EDX spectra of A2-PCL and S2-PCL materials before and after incubation in SBF, as well as DMEM-10\% FBS for 14 days. PCL film did not reveal any morphological and chemical changes after incubation in $\mathrm{SBF}$, while the surface of that material after exposure to DMEM-10\% FBS was enriched with a small amount of calcium (Ca) and phosphorus (P), without significant changes in surface morphology. In turn, the surfaces of A2-PCL and S2-PCL composites after 14-day incubation in both media were covered with the layers rich in $\mathrm{Ca}$ and $\mathrm{P}$, what was confirmed by EDX analysis. All of the layers exhibited spherical cauliflower-like morphologies, typical of carbonated hydroxyapatite (HCA) [21]. However, layer morphologies varied between materials incubated in SBF and DMEM-10\% FBS-spherical forms developed in cell culture medium were significantly smaller compared to that formed in SBF. In the case of composites containing A2 glass particles, the lack of silicon (Si) derived from BG confirmed that the layers were thick and uniform. Furthermore, on the surface of $21 \mathrm{~A} 2<45 \mu \mathrm{m}-\mathrm{PCL}$ composite multilayer deposits can be observed after incubation in both media. In turn, a small amount of Si was still detected on the 


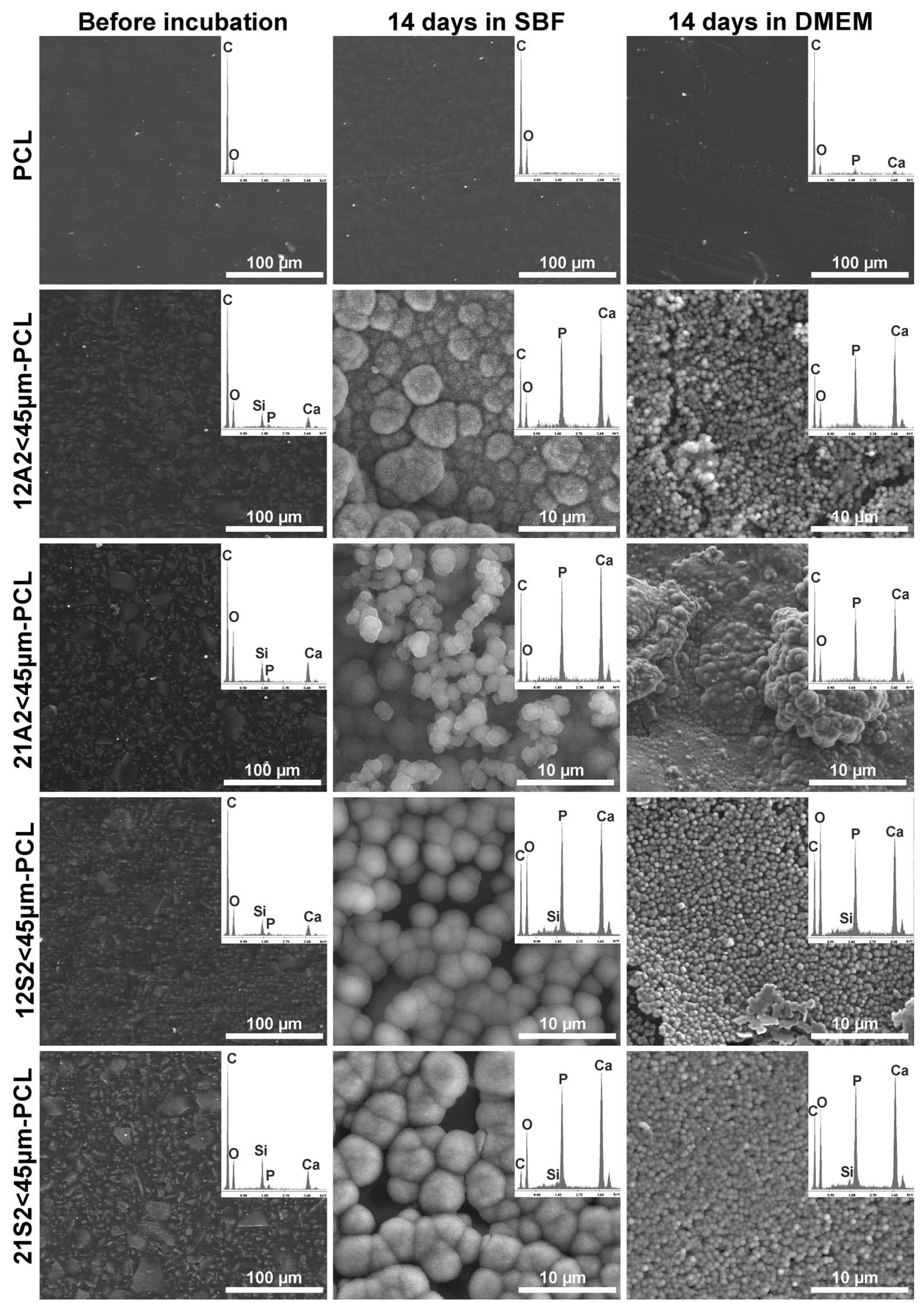


4 Figure 1 SEM images and EDX spectra (averaged for the entire analysed surface) of GS surfaces of the films before and after 14-day incubation in SBF and DMEM-10\% FBS.

surfaces of S2-PCL films after immersion in SBF and DMEM-10\% FBS, indicating lower thickness/tightness of calcium phosphate $(\mathrm{CaP})$ layers and/or formation of silica-rich layer directly on the surface of composites.

Figure 2 shows ATR-FTIR spectra of the obtained materials before and after 14-day incubation in SBF and DMEM-10\% FBS. No significant changes indicating the formation of $\mathrm{CaP}$ layer on the surface of PCL film after 14-day incubation in both media were found. FTIR spectra of all composites exhibited appearance of new two bands in the range of $560-602 \mathrm{~cm}^{-1}$ and single intensive band at $1020 \mathrm{~cm}^{-1}$ originated from $\mathrm{CaP}$ layer after 14 days of soaking. $\mathrm{New}$ bands can be assigned to $\mathrm{O}-\mathrm{P}-\mathrm{O}$ bending mode and $\mathrm{P}-\mathrm{O}$ stretching mode, respectively. Furthermore, new absorption band corresponding to the carbonate groups $\mathrm{CO}_{3}{ }^{2-}$ in HCA was found at $873 \mathrm{~cm}^{-1}$ (outof-plane bending mode) [22]. In the case of A2-PCL composite films, the almost complete vanishing of bands characteristic for PCL was observed after incubation, confirming high thickness and uniformity of the formed CaP layer. FTIR spectra of S2-PCL films after incubation in DMEM-10\% FBS and SBF exhibited more narrow and sharp distribution of the band at $1020 \mathrm{~cm}^{-1}$ compared to spectra registered for A2PCL films, indicating probably slightly higher crystallinity of calcium phosphate layer.

The variations in $\mathrm{Ca}, \mathrm{P}$ and $\mathrm{Si}$ concentrations in $\mathrm{SBF}$ and DMEM-10\% FBS during film incubation are shown in Fig. 3a-c. The kinetics of $\mathrm{Ca}, \mathrm{P}$ and $\mathrm{Si}$ changes depended on content and chemical composition of bioactive glass particles, as well as type of incubation medium. In the case of PCL film, Ca and P concentration in SBF did not change significantly over soaking time, while in culture medium their content decreased gradually. For composite materials, Ca concentration was affected by both its release from the glass structure and its uptake resulting from calcium phosphate layer formation on the material surfaces [23]. The Ca release/consumption profiles were markedly different for SBF and DMEM-10\% FBS (Fig. 3a). Ca content in SBF gradually increased at the day 1 for $12 \mathrm{~S} 2<45 \mu \mathrm{m}-\mathrm{PCL}$ and up to day 7 for both A2-PCL composites and 21S2 $<45 \mu \mathrm{m}-\mathrm{PCL}$

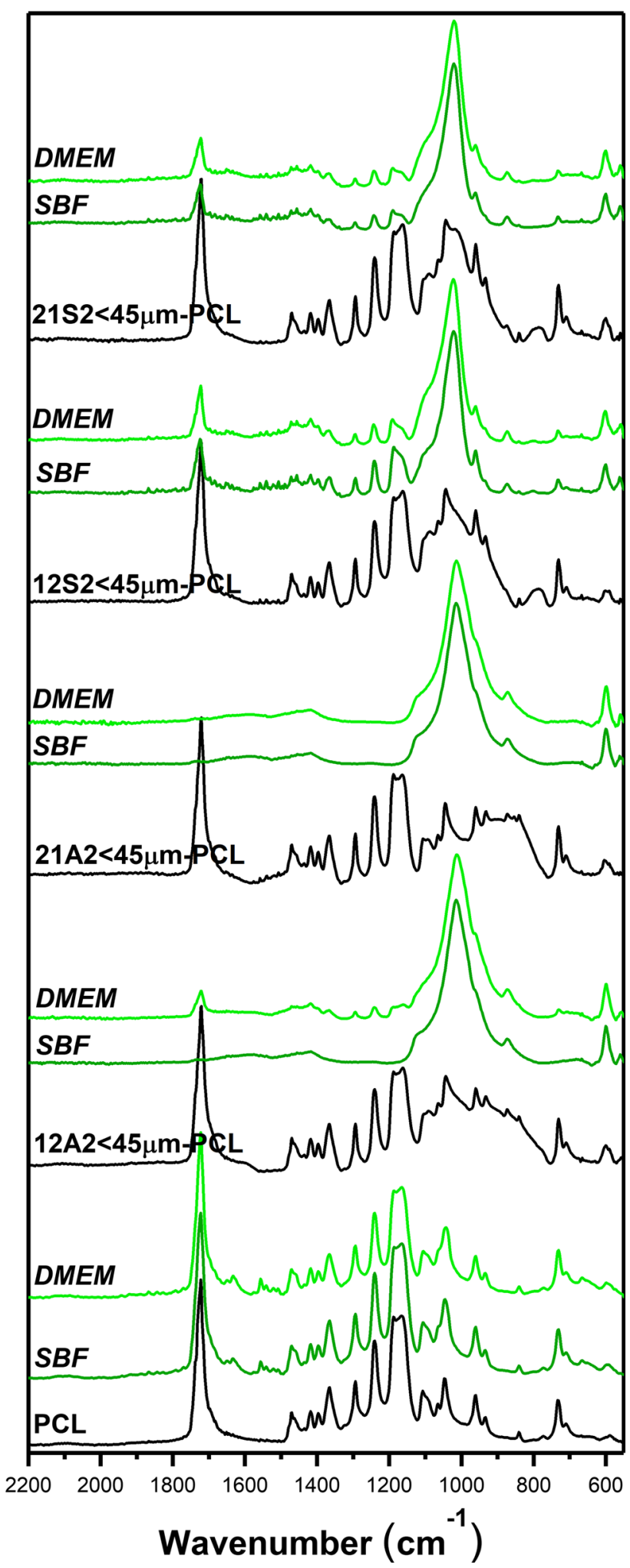

Figure 2 ATR-FTIR spectra of GS surfaces of the films before and after 14-day incubation in SBF and DMEM-10\% FBS. 

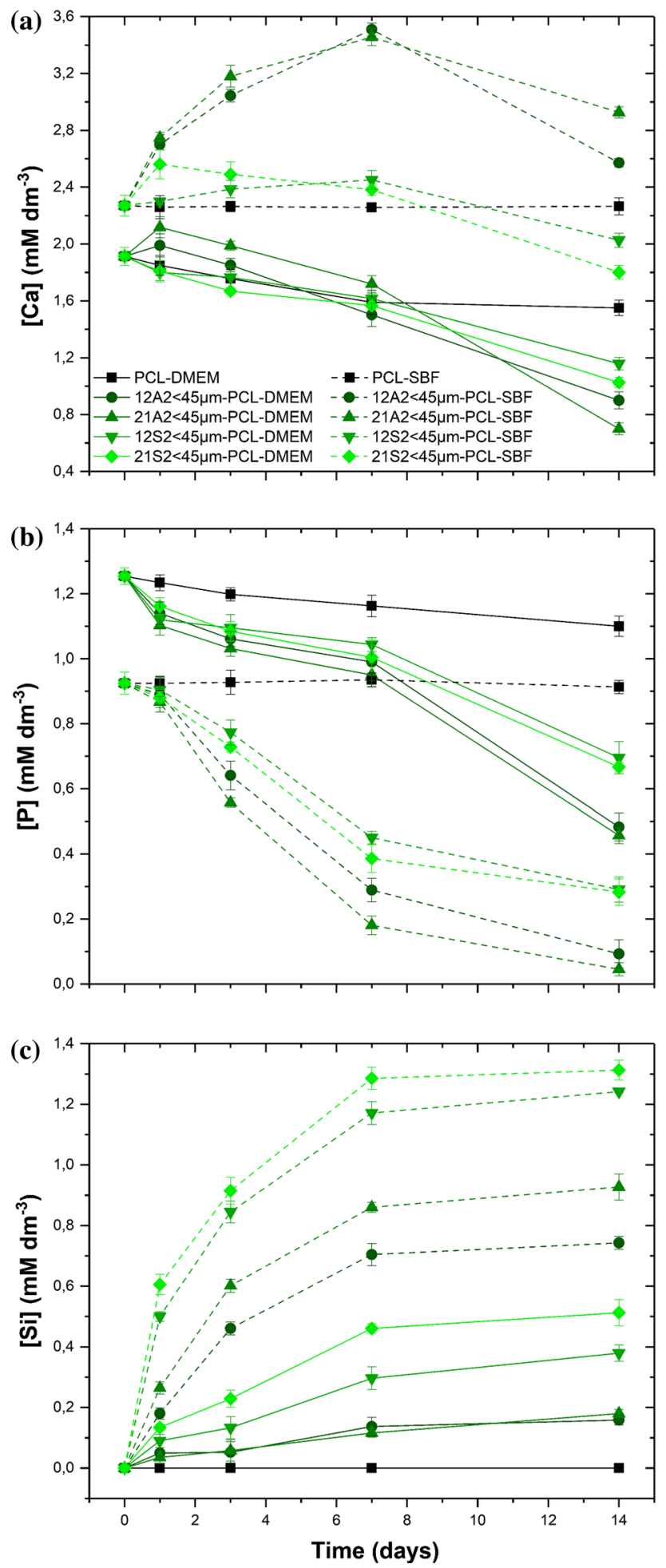

Figure 3 Changes in $\mathrm{Ca}(\mathbf{a}), \mathrm{P}$ (b) and $\mathrm{Si}$ (c) concentrations in SBF and DMEM-10\% FBS during 14-day soaking.

film; after that, the Ca content gradually decreased. It can be observed that the release rate of Ca to SBF from A2-PCL materials was significantly higher compared to films containing S2 BG, which is directly related to higher concentration of $\mathrm{CaO}$ in $\mathrm{A} 2$ glass structure. Up to day 7, the decrease in Ca concentrations, resulting from ion uptake caused by formation of $\mathrm{CaP}$ layer, was compensated by high release rate of $\mathrm{Ca}$ from $\mathrm{A} 2 \mathrm{BG}$. The depletion of $\mathrm{Ca}$ between day 7 and 14 resulted from $\mathrm{CaP}$ layer formation, what additionally inhibited further $\mathrm{Ca}$ release from the materials. These changes correlate with $\mathrm{Si}$ concentration profiles, for which Si release was significantly reduced after 7 days of incubation (Fig. 3c). In turn, Ca content in DMEM-10\% FBS increased only at day 1 for A2-PCL composites; however, the changes were significantly lower than in SBF. Furthermore, film containing 21 vol\% A2 glass particles released more $\mathrm{Ca}^{2+}$ ions compared to composite with $12 \mathrm{vol} \%$ A2 fillers. In the case of PCL and S2-PCL films, Ca concentration gradually decreased from the first day of incubation. The highest depletion of Ca in DMEM-10\% FBS for all composites was observed from day 7 to day 14 , what can be attributed to $\mathrm{CaP}$ layer formation. The decrease in P concentration in SBF and DMEM-10\% FBS confirmed CaP layer deposition on the surfaces of composite films (Fig. $3 \mathrm{~b}$ ). However, the $\mathrm{P}$ consumption profiles for both of the incubation media showed different trends. In the case of cell culture medium, the fastest decrease in $\mathrm{P}$ concentration can be observed at day 1 and between day 7 and 14, while in SBF the highest reduction was observed from day 1 to 7. It can be observed that the reduction in P content in SBF and DMEM-10\% FBS for A2-PCL composites was higher compared to S2-PCL films.

\section{In vitro osteoblast response}

Normal human osteoblasts were cultured in direct contact with PCL film, as well as A2-PCL and S2-PCL composites, containing 12 and $21 \mathrm{vol} \%$ glass particles of $<45 \mu \mathrm{m}$ size. As shown in Fig. $4 \mathrm{a}$, a significant increase in the number of intact adherent cell between 7 and 14 days of culture on all tested materials has been observed. Furthermore, osteoblasts just after 7-day culture were uniformly distributed on the surfaces of all films, spread well and showed a polygonal morphologies (Fig. 4e). After 7 days, composite films showed similar number of cells; however, the values were significantly lower compared to TCPS and simultaneously higher in comparison with PCL material. After 14 days, 

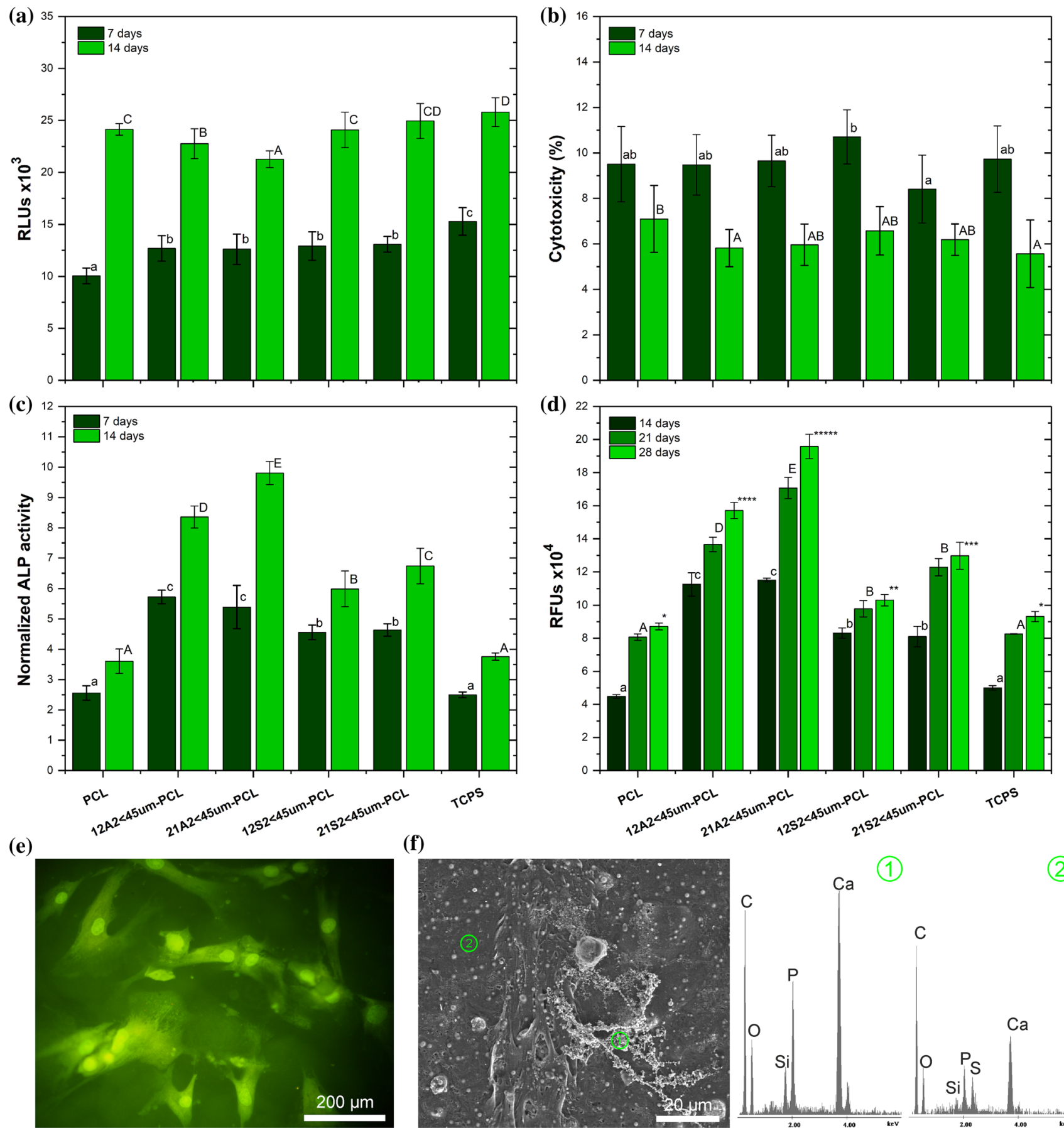

(f)
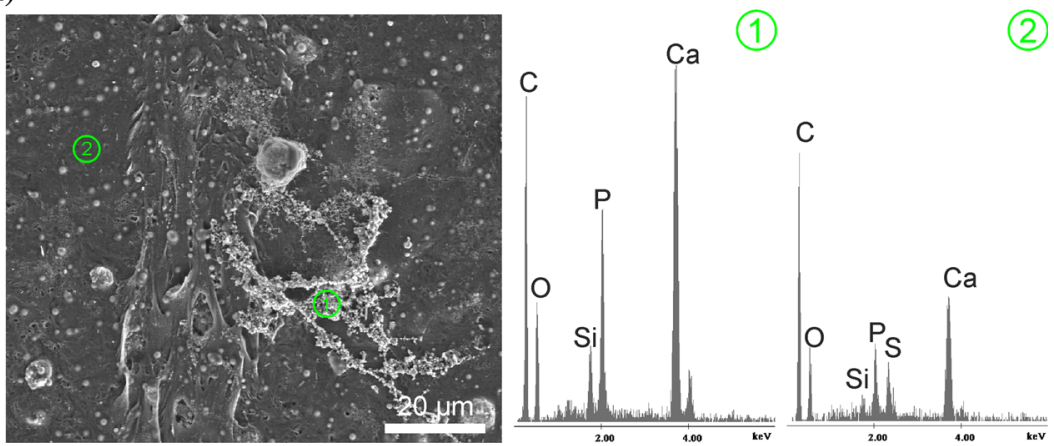

Figure 4 Adenylate kinase (AK) level in the lysate corresponding to the number of intact adherent cells (a), AK level in the supernatant related to AK level in the lysate representing material cytotoxicity (b), ALP activity normalized to cell number (c), ECM mineralization (d). Results are expressed as mean \pm SD. Statistically significant differences $(p<0.05)$ between each film and

number of cells seeded on composites containing A2 glass particles was significantly lower compared to other films and control material (TCPS).
TCPS after different cell culture periods are indicated by upper and lower case, as well as asterisks, respectively. Fluorescence microscopy image of osteoblasts grown on the $21 \mathrm{~A} 2<45 \mu \mathrm{m}$ PCL composite for 7 days (e), SEM image and EDX spectra of a osteoblast layer on the $21 \mathrm{~A} 2<45 \mu \mathrm{m}$-PCL film after 28 days of culture (f).

Cytotoxicity of obtained films, estimated based on the measurement of adenylate kinase (AK) released from the damaged cells, is shown in Fig. $4 \mathrm{~b}$. 
Cytotoxicity of all the films decreased over time and did not exceed $11 \%$ after 7 days and $7 \%$ after 14 days. After both culture periods, no significant differences were found between all obtained materials and TCPS, indicating no cytotoxic effects on osteoblasts.

Alkaline phosphatase (ALP) activity, an early marker of osteoblast differentiation, was measured after 7 and 14 days of culture. The results were presented as ALP activity normalized to cell number (Fig. 4c). After 7 and 14 days of culture, osteoblasts seeded on composites exhibited significantly higher ALP activity compared to cells in contact with PCL film and TCPS, for which the values were on similar level. Osteoblasts cultured on both of the A2-PCL films showed the highest ALP activity. These early differentiation characteristics can be correlated with the decrease in proliferation rate after 14 days of culture [24]. After 7 days, no statistically significant differences between films containing different content of A2 particles were observed, while after 14 days, the values recorded for $21 \mathrm{~A} 2<45 \mu \mathrm{m}-\mathrm{PCL}$ were significantly higher. Materials containing S2 particles showed similar dependency.

For all films and control material (TCPS), extracellular matrix (ECM) mineralization increased with increasing culture time; however, the greatest changes were observed for materials containing $21 \mathrm{vol} \%$ glass particles, especially for 21A2 $<45 \mu \mathrm{m}$-PCL film (Fig. 4d). Osteoblasts cultured on composite materials showed significantly higher level of mineralization compared to cells seeded on PCL film and TCPS. As in the case of ALP activity, cells seeded on both of the A2-PCL films exhibited the highest ECM mineralization. After 14 days, no statistically significant differences between films containing different content of A2 particles were observed, while after 21 and 28 days, the values recorded for $21 \mathrm{~A} 2<45 \mu \mathrm{m}-\mathrm{PCL}$ were significantly higher. The same trend was observed for S2-PCL composite films. ECM mineralization process was also confirmed with SEM/EDX analysis (Fig. 4f). After 28 days of culture, a confluent layer of osteoblasts (confirmed by the presence of sulphur in EDX analysis) covered with mineral deposits rich in calcium and phosphorus was observed on the surface of the $21 \mathrm{~A} 2<45 \mu \mathrm{m}$-PCL film.

\section{In vitro degradation}

A2-PCL and S2-PCL composite films, containing 21 vol $\%$ glass particles of $<45$ and $<3 \mu \mathrm{m}$ sizes, as well as pure PCL material were subjected to long-term hydrolytic degradation test by incubation in PBS solution for 1, 3, 6, 9, 12 and 15 months. Composites with $21 \mathrm{vol} \%$ glass particles were selected for longterm degradation studies because of their excellent in vitro bioactivity and also promising results of in vitro biological studies presented in previous sections. Furthermore, our previous results [25, 26] showed that glass particle size $(<45$ and $<3 \mu \mathrm{m})$ influence osteoblast response in contact with PCLbioactive glass membranes, as well as in vitro bioactivity of membranes and films. Therefore, degradation behaviour of composites with fillers of different sizes is also considered in this work. Figure 5 shows SEM images and EDX spectra of AS and GS surfaces of A2-PCL and S2-PCL materials before and after 6and 15-month incubation in PBS. The PCL film immersed in PBS showed the surface morphologies similar to those not degraded. The entire surfaces of PCL material were just enriched with sodium $(\mathrm{Na})$ and chloride $(\mathrm{Cl})$ and also covered with several $\mathrm{NaCl}$ precipitations. On both AS and GS surfaces of $21 \mathrm{~A} 2<3 \mu \mathrm{m}-\mathrm{PCL}$ and $21 \mathrm{~S} 2<3 \mu \mathrm{m}-\mathrm{PCL}$ films, the layers rich in $\mathrm{Ca}$ and $\mathrm{P}$ with small amount of $\mathrm{Na}$ and $\mathrm{Cl}$ were formed during immersion in PBS. After 6 months, the layers on both materials showed uniform morphology. In the case of $21 \mathrm{~S} 2<3 \mu \mathrm{m}-\mathrm{PCL}$ material, no significant changes were observed over incubation time, while the morphology of layers formed on the 21A2 $<3 \mu \mathrm{m}$-PCL film evolved to fine poorly developed spherical forms. In contrast to composites containing glass particles of $<3 \mu \mathrm{m}$ size, both surfaces of the materials with bigger fillers $(<45 \mu \mathrm{m})$ showed different behaviour upon immersion in PBS. AS surface of $21 \mathrm{~A} 2<45 \mu \mathrm{m}$-PCL film after immersion in PBS was covered with porous $\mathrm{CaP}$ layer. The morphology did not change over incubation time and indicated its amorphous nature. In turn, on GS surface thick CaP layer with fine spherical aggregates has developed. No significant morphological changes in AS surface of $21 S 2<45 \mu \mathrm{m}$ PCL material were observed up to 15 months. The surface was just enriched with small amount of $\mathrm{Na}$ and $\mathrm{Cl}$. In the case of GS surface, $\mathrm{CaP}$ layer formation occurred; however, concentration of $\mathrm{Ca}$ and $\mathrm{P}$ was significantly higher in the regions of films, where 


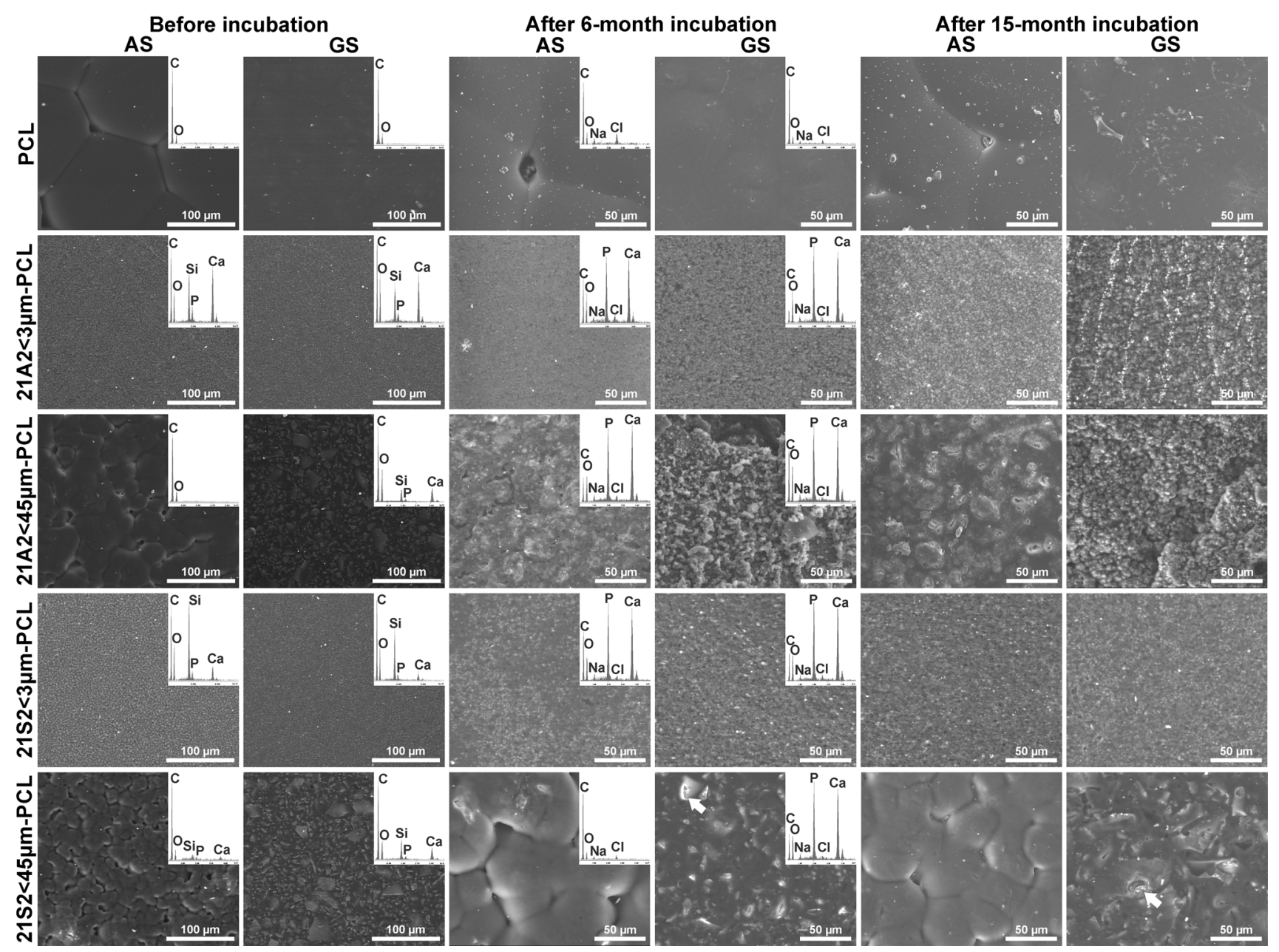

Figure 5 SEM images and EDX spectra (averaged for the entire analysed surface or collected at the point indicated by the arrow) of both GS and AS surfaces of the films before and after 6- and 15-month incubation in PBS.

glass particles were visible (indicated by arrow). The morphology of formed layer once more suggested its amorphous nature. The lack or small amount of $\mathrm{Si}$ derived from BG indicated a large thickness and uniformity of the layers formed on the surfaces of films. The results suggested that the deposition occurred significantly faster and to a greater extent on the surfaces of 21A2-PCL composites compared to 21S2-PCL films.

The changes in PBS pH values during the incubation of materials are shown in Fig. 6a. It can be seen that $\mathrm{pH}$ values for PCL and both S2-PCL materials stayed within the physiological range, and varied between 7.3 and 7.4 during the whole incubation period. In the case of A2-PCL composites, the $\mathrm{pH}$ values showed a rapid increase in the first month of immersion and reached 8.22 for $21 \mathrm{~A} 2<45 \mu \mathrm{m}-\mathrm{PCL}$ film and 8.46 for $21 \mathrm{~A} 2<3 \mu \mathrm{m}-\mathrm{PCL}$. After that time,
$\mathrm{pH}$ values decreased; however, the reduction was much faster in the case of $21 \mathrm{~A} 2<3 \mu \mathrm{m}$-PCL composite, for which $\mathrm{Ca}$ initial level was regained after 3 months, while for $21 \mathrm{~A} 2<45 \mu \mathrm{m}$-PCL film $\mathrm{pH}$ value returned to physiological range after 6 months.

Degradation process was also monitored by measuring mass loss of the materials during incubation in PBS (Fig. 6b). PCL film showed an increase in mass during the whole incubation period, what was likely associated with $\mathrm{NaCl}$ deposition. After 3 months of incubation, mass increase reached about 5\% and stayed on similar level until the end of the test. In the case of both 21A2-PCL composites, mass has also increased during the first month; however, higher increase was noted for films containing glass particles of $<3 \mu \mathrm{m}$ size. After 3-month immersion, $21 \mathrm{~A} 2<3 \mu \mathrm{m}-\mathrm{PCL}$ film still showed increase in mass, however, significantly lower than after first month. 

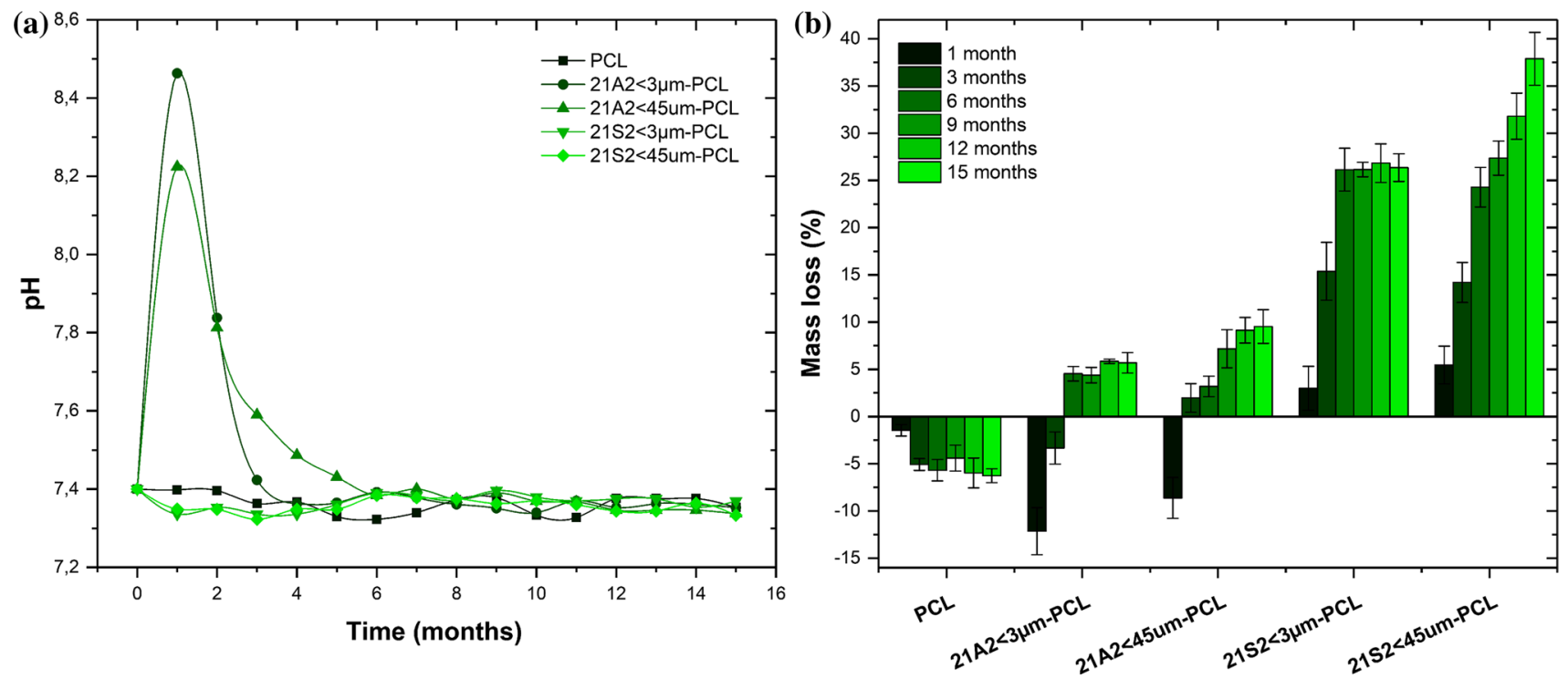

Figure $6 \mathrm{pH}$ changes of PBS (a) and mass loss of the films (b) during 15-month incubation.

These changes were related to $\mathrm{CaP}$ layer formation. From month $6,21 \mathrm{~A} 2<3 \mu \mathrm{m}$-PCL composite showed mass reduction (about 5\%) that did not change until the end of the incubation. 21A2 $<45 \mu \mathrm{m}$-PCL material began to lose its mass at month 3 , and the reduction had proceed gradually up to month 15 , reaching values of about $10 \%$. In the case of $21 \mathrm{~S} 2-\mathrm{PCL}$ composites, no mass increase was observed. Both 21S2-PCL composites showed significantly greater mass loss in comparison with 21A2-PCL films. The highest mass reduction was observed at month 3 and 6 for both 21S2-PCL composites. Mass reduction of $21 \mathrm{~S} 2<3 \mu \mathrm{m}$-PCL film was stopped at month 6 and stabilized at the level of about $26 \%$ up to 15 month. In turn, $21 \mathrm{~S} 2<45 \mu \mathrm{m}-\mathrm{PCL}$ composite exhibited gradual increase in mass loss, reaching values of about $38 \%$ at the end of the test.

The changes in the degree of crystallinity $\left(\chi_{c}\right)$ and the melting temperature $\left(T_{\mathrm{m}}\right)$ of obtained films before and after each incubation period, estimated based on the DSC analysis, are shown in Fig. 7a-c. As have been shown in our previous works [19, 26], the presence of bioactive glass particles in polymer matrix reduces its crystallinity and melting temperature. Significantly higher reduction was found for composites containing glass particles of $<45 \mu \mathrm{m}$ size. The decrease in $\chi_{\mathrm{c}}$ and $T_{\mathrm{m}}$ indicates hindering effect of glass fillers on crystallization process of PCL, caused by the limitation of polymer chain mobility and therefore the reduction in ability to rearrange into well-ordered regions. For all of the materials, the degree of crystallinity and melting temperature of PCL matrix increased with incubation time in PBS. In the case of semicrystalline polymers, such as PCL, the hydrolysis occurs preferentially in amorphous regions, inducing removal of shorter polymer chains. Therefore, an increase in the crystallinity fraction of polymer over degradation time was expected. Moreover, increase in $T_{\mathrm{m}}$ during incubation can also indicate recrystallization process of PCL and a formation of larger polymer crystallite structure [27, 28]. The hydrolysis of polymer chains increases possibility of recrystallization by reducing their length and increasing mobility. Furthermore, because of low glass transition temperature of PCL $\left(-60{ }^{\circ} \mathrm{C}\right)$ mobility of non-degraded chains at $37^{\circ} \mathrm{C}$ during incubation in aqueous media could be enhanced and solvent-induced crystallization might have occurred [8, 28-30]. Although the crystallinity of PCL film increased, the mass loss was not observed, indicating bulk degradation kinetics. This is in good agreement with results obtained by Lam et al. [8]. In the case of composites, no further changes or even decrease in $\mathrm{T}_{\mathrm{m}}$ after 15 months was observed, suggesting that hydrolysis extended to crystalline regions of PCL [28]. As shown in Fig. 7b, composites containing glass particles of $<45 \mu \mathrm{m}$ size exhibited higher increase in polymer matrix crystallinity during incubation compared to films with smaller particles $(<3 \mu \mathrm{m})$. When considering chemical composition of glass, greater increase in $\chi_{c}$ was observed for S2-PCL materials. The biggest changes in matrix crystallinity 

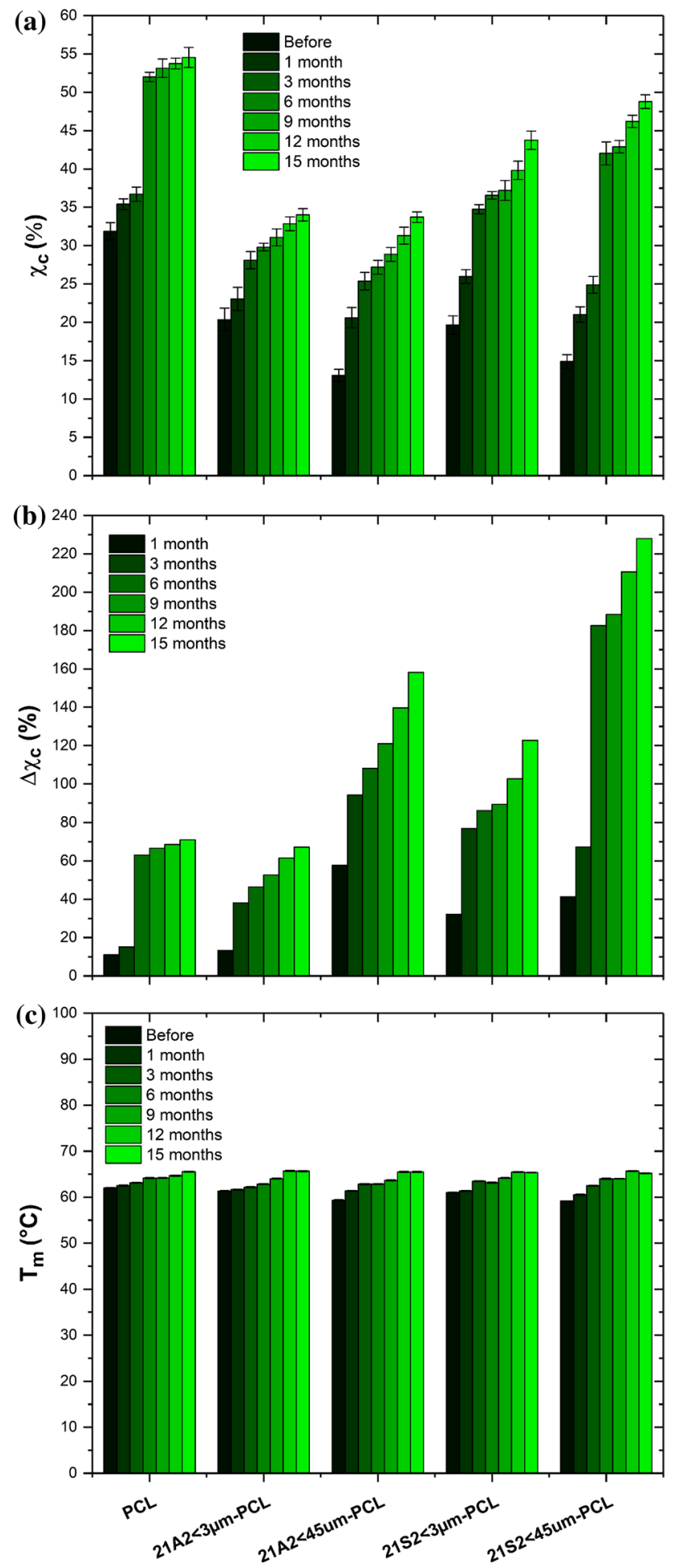

Figure 7 Degree of crystallinity (a), percentage of changes in crystallinity (b) and melting temperature (c) of PCL matrix during 15-month incubation in PBS.

were noted in the first three months for 21A2 $<3 \mu \mathrm{m}$ PCL,$\quad 21 \mathrm{~A} 2<45 \mu \mathrm{m}-\mathrm{PCL}$ and $21 \mathrm{~S} 2<3 \mu \mathrm{m}-\mathrm{PCL}$ films, as well as first six months for $21 \mathrm{~S} 2<45 \mu \mathrm{m}$ PCL composite.

The changes in the tensile strength $\left(\sigma_{\mathrm{M}}\right)$ and the Young's modulus $\left(E_{t}\right)$ of obtained films before and after each incubation period are shown in Fig. 8a, b. According to results obtained previously [26], the addition of glass particles of $<45 \mu \mathrm{m}$ size into polymer matrix resulted in significant reduction in $\sigma_{\mathrm{M}}$, while smaller particles $(<3 \mu \mathrm{m})$ did not change the tensile strength of the films significantly. Composites containing glass particles of both sizes showed significantly higher Young's modulus compared to PCL films; however, the smaller fillers $(<3 \mu \mathrm{m})$ increased $E_{\mathrm{t}}$ more effectively than particles of $<45 \mu \mathrm{m}$ size. The tensile strength of all films decreased gradually with increasing degradation time; however, the highest reduction was noted in the first month of incubation. Composite materials showed significantly greater decrease in $\sigma_{\mathrm{M}}$ than PCL film. Young's modulus of PCL film increased gradually during incubation, while all composite films showed reduction in $\mathrm{E}_{\mathrm{t}}$. Increase in stiffness of PCL film can be attributed to an increase in degree of crystallinity [30, 31]. In the case of A2-PCL composites, the biggest changes in $E_{\mathrm{t}}$ were noted in the first 3 months, and then, the values stayed on similar level until the end of the test. In contrast, the highest reduction in Young's modulus of S2-PCL films was observed in the first month, after which the values have been still decreasing, however, more gradually. Composites containing S2 glass particles exhibited higher reduction in tensile strength, as well as in Young's modulus during incubation compared to A2-PCL materials, while particle size seemed to have no significant influence on mechanical parameters during incubation in PBS.

\section{Discussion}

Materials for bone tissue engineering should possess controllable degradation kinetics and also the ability to support and actively promote bone repair and regeneration, depending on target application. In present work, we have proved that long-term degradation behaviour, bone bonding ability and also osteoinductive properties of poly( $\varepsilon$-caprolactone)based composites could be controlled and optimized by using gel-derived bioactive glass fillers with different contents, sizes and chemical compositions. 

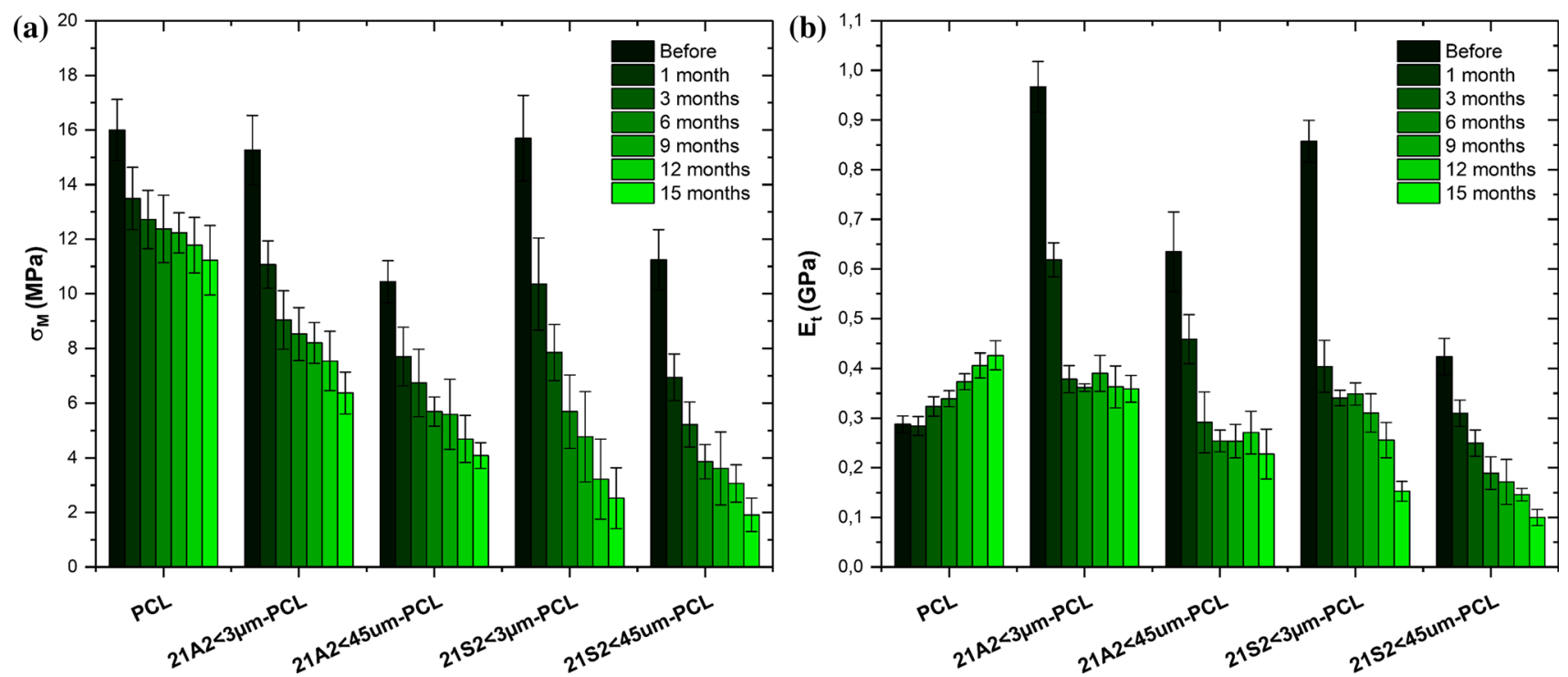

Figure 8 Tensile strength (a) and Young's modulus (b) of the films during 15-month incubation in PBS.

\section{In vitro bioactivity}

The differences in ion release/consumption profiles and also morphologies of formed layers between SBF and DMEM-10\% FBS suggest differences in the conditions of the layer formation between these two media which can be explained by the presence of proteins and amino acids in DMEM, supplemented additionally with $10 \%$ FBS, in contrast to SBF containing only inorganic components. The adsorption of proteins from body fluids and cell culture medium onto the material surface occurs as one of the first events when the biomaterial is implanted into the body or soaked in the medium [32]. On the one hand, it was previously demonstrated that the in vitro calcium phosphate layer forming ability of bioactive ceramics coated with proteins was reduced [33]. The layer of organic matter on the surface of bioactive material creates a barrier that inhibits dissolution and exchange of the ions-the first and crucial step of the bioactive process, which can be confirmed by significant reduction in $\mathrm{Si}$ release (Fig. 3c) and also immediate decrease in Ca concentration (Fig. 3a) in DMEM-10\% FBS compared to SBF. What is more, nucleation of calcium phosphates can be inhibited by the Ca-chelating properties of the biological compounds present in cell culture medium, which cause a decrease in the supersaturation state of the solution [34]. On the other hand, proteins and amino acids adsorbed on material surface may represent effective binding sites for $\mathrm{Ca}^{2+}$ and $\mathrm{PO}_{4}{ }^{3-}$ ions. Ion binding capacity depends on acidity/basicity and electric charge of proteins and amino acids, which are govern by properties of biomaterial surface (chemical composition, wettability, zeta potential and topography), properties of adsorbed organic molecules (type, size, charge and conformation), as well as solution $\mathrm{pH}$ (affecting by dissolution behaviour of the material) [35]. Therefore, heterogeneous nucleation may be enhanced by the adsorbed organic molecules, which can create necessary crystal nucleation sites on the surface [34]. An uniform layer of proteins and amino acids on the surfaces of composite films incubated in DMEM-10\% FBS probably led to higher nucleation density of calcium phosphates and therefore development of significantly smaller crystallites compared to incubation in SBF (Fig. 1). Furthermore, the differences in kinetics of $\mathrm{P}$ consumption between DMEM-10\% FBS and SBF at the first day of incubation can indicate different mechanisms of $\mathrm{CaP}$ nucleation between these two media (Fig. 3b). It seems that in DMEM-10\% FBS, $\mathrm{Ca}^{2+}$ and $\mathrm{PO}_{4}{ }^{3-}$ ions were adsorbed simultaneously on the surface of composites covered with proteins, while in SBF the first stage of nucleation was accumulation of $\mathrm{Ca}^{2+}$ ions which then attracted phosphate groups. In that case, CaP nucleation probably occurred on the surface of glass particles present on the GS surface of composites in accordance with mechanism of bioactivity proposed by Kokubo and Hench [36]. It was previously shown that the layers of proteins and amino acids on the surfaces of graphene oxide and 
polymers (PS, PMMA) induced the nucleation of hydroxyapatite in SBF and 1.5SBF [37, 38], which can explain the enrichment of pure PCL film surface with $\mathrm{Ca}$ and $\mathrm{P}$ (Fig. 1) with an accompanying decrease in $\mathrm{Ca}$ and $\mathrm{P}$ concentration in DMEM-10\% FBS (Fig. 3a, b).

The results clearly show the differences in kinetics of calcium phosphate layer formation on the surfaces of composite films depending on the content (12 and $21 \mathrm{vol} \%$ ) and the chemical composition (A2 and S2) of bioactive glasses. Dependence between in vitro bioactivity and the content of bioactive glass particles in polymer matrix of composite scaffolds was previously reported [39, 40]. In our previous works, we have shown that calcium phosphate layer forming ability depends on chemical composition of bioactive glasses [41, 42] and also on their particle size [25, 26]. Our previous results showed that the increasing content of $\mathrm{CaO}$ in $\mathrm{SiO}_{2}-\mathrm{CaO}-\mathrm{P}_{2} \mathrm{O}_{5}$ gel-derived glasses results in higher $\mathrm{Ca}$ dissolution from glass structure [43]. Therefore, dissolution of $\mathrm{Ca}^{2+}$ ions from A2-PCL composites was more effective than from S2-PCL films, leading to faster supersaturation of SBF and DMEM-10\% FBS and therefore faster nucleation and crystallization of calcium phosphate layer. In summary, our current and past research proved that the use of different content, chemical composition and particle size of bioactive glass fillers is a simple way to modulate bioactivity of polymer-based composites.

\section{In vitro osteoblast response}

Obtained results indicate that poly( $\varepsilon$-caprolactone)/ bioactive glass composites stimulate osteoblast proliferation, differentiation and also matrix mineralization. Furthermore, it seems that osteoblast response depends on both chemical composition of glass particles and their content in polymer matrix. These differences can be explained by different kinetics of $\mathrm{Ca}$ and Si release form composite films to culture medium, as was revealed in ICP-OES analysis of DMEM (Fig. 3). Several in vitro studies showed that the osteoblast response was significantly improved upon addition of bioactive glass fillers, including particles and fibres, into polymer matrix [44, 45]. Kim et al. [44] exhibited that collagen synthesis, ALP activity and mineralization of the murinederived osteoblast (MC3T3-E1) cultured on poly(D,Llactic acid)/bioactive glass nanocomposites increased with increasing content of nanofibre glass in polymer matrix. Furthermore, it was shown that the ionic products of bioactive glasses stimulate the expression of genes of osteoblastic cells, which in turn modulates osteogenesis and promote bone formation [13]. Yao et al. [46] noted that the dissolution products from poly(lactic-co-glycolic)/bioactive glass composite scaffold stimulated osteogenesis of rat marrow stromal cells, as was confirmed by increased mRNA expression of osteoblastic markers such as osteocalcin, alkaline phosphatase and bone sialoprotein. In another work [47], human primary osteoblast-like cells cultured in contact with melt-derived 45S5, as well as gel-derived $58 \mathrm{~S}$ and $77 \mathrm{~S}$ bioactive glasses revealed different behaviour, including ALP activity, as well as collagen type I expression and synthesis. The authors pointed out that the 45S5 glass significantly increased collagen secretion and ALP activity, compared to the other two gel-derived bioactive glasses, which can be related to alkalization of the culture medium. It was previously shown that an increase in $\mathrm{pH}$ induced by calcium release from glass structure has a significant effect on the expression of collagen type I, the crosslinking of the collagen chains and the subsequent precipitation of hydroxyapatite, as well as cellular metabolism and genes important for osteoblastic functions [47, 48]. Therefore, the highest ALP activity and ECM mineralization of the osteoblasts cultured on A2-PCL composites (Fig. 4c, d) can be correlated with an increase in $\mathrm{Ca}^{2+}$ concentration and also cell culture alkalization, confirmed in bioactivity (Fig. 3a) and degradation (Fig. 6a) tests. What is more, our previous results demonstrated that the addition of A2 and S2 bioactive glass particles into PCL matrix improved nanoand microroughness and also enhanced the hydrophilicity of composite GS surfaces [19], which can also affect osteoblast response. It was shown that osteoblast behaviour is greatly influenced by surface properties, including hydrophilicity, roughness, texture and morphology [49].

\section{In vitro degradation}

In the literature, ceramic fillers, including bioactive glass particles, affected degradation behaviour of aliphatic polyesters in two ways, depending on polymer crystallinity, and thus its degradation rate. In the case of amorphous, fast degrading polymers, such as poly(DL-lactide) (PDLLA) and poly(lactideco-glycolide) (PLGA), degradation with random 
chain scission by ester hydrolysis in a process autocatalysed by the generation of carboxylic acid end groups occurs. The dissolution of alkaline ions (e.g. $\mathrm{Ca}^{2+}, \mathrm{Na}^{+}$) from the bioactive glass structure can delay degradation of polymer matrix by neutralizing the released acidic by-products and thus prevent autocatalytic action $[9,50]$. On the other hand, modification of semicrystalline polymers (e.g. PCL) with hydrophilic ceramic fillers, such as nano-HAp [10], TCP [8], bioactive glass and calcium silicate particles [11], improved surface wettability, enhanced water absorption and diffusion into the bulk of polymer matrix, and also increased the surface area of a hydrolytic attack through the interface of the fillers and polymer. This, in turn, led to faster polymer degradation. Furthermore, the presence of ceramic filler in polymer matrix resulted in reduction in its initial degree of crystallinity, making them more susceptible for degradation [8]. The results obtained here agreed with these findings and clearly showed that the modification of PCL with bioactive glass particles accelerates its degradation. Furthermore, we proved that long-term degradation behaviour of PCL was significantly modified depending on particle size and chemical composition of bioactive glass fillers. It was previously shown that the smaller particles of bioactive glass, incorporated into poly( $\varepsilon$-caprolactone-co-DL-lactide) matrix, significantly enhanced the water absorption compared to larger particles, resulting in faster loss of polymer molecular weight [9]. In the literature, there are several works indicating that the chemical composition of resorbable phosphate glasses significantly affects degradation of PCL matrix [31, 51]. Phosphate glass fillers, depending on formulation, dissolve in different rate, creating voids and channels within the polymer matrix, affecting water absorption, and therefore polymer degradation. However, there are limited research works reporting the effect of chemical composition of slow degrading silicate bioactive glasses on degradation behaviour of polymer matrix.

In contrast to the findings discussed above, higher mass loss (Fig. 6b) and increase in polymer matrix crystallinity (Fig. 7b), during incubation in PBS, suggested that degradation of composites with larger-sized glass particles $(<45 \mu \mathrm{m})$ proceed faster compared to films with smaller ones $(<3 \mu \mathrm{m})$. In turn, higher reduction in mass (Fig. 6b), tensile strength (Fig. 8a) and Young's modulus (Fig. 8b), as well as increase in degree of crystallinity (Fig. 7b), during incubation in PBS indicated higher degradation rate of the composites containing S2 bioactive glass particles compared to A2-PCL films. Our findings would appear to suggest that the degradation of PCL-bioactive glass composites was governed mainly by calcium phosphate layer forming ability on their surfaces. CaP layer, formed on surface of composites upon immersion in PBS, seems to play a moderating role in composite degradation, protecting polymer matrix against direct exposition to aqueous environment. The greatest reduction in mass and mechanical parameters, as well as the highest increase in degree of crystallinity at the beginning of incubation in PBS, when the CaP layer has already been forming, would appear to confirm this conception.

In contrast to SBF, phosphate buffer solution does not contain $\mathrm{Ca}^{2+}$ ions and, however, is rich in $\mathrm{PO}_{4}{ }^{3-}$ species. Therefore, abilities of both PCL-A2 and PCLS2 composites to form calcium phosphate layer in PBS confirm that both glasses are a sufficiently rich source of calcium ions for obtaining supersaturation of PBS and calcium phosphate layer formation, however, in different rates. Mass increase in the first months of incubation (Fig. 6b) as well as SEM and EDX (Fig. 5) results indicated that the deposition of $\mathrm{CaP}$ layer occurred significantly faster and to a greater extent on the surfaces of 21A2-PCL composites compared to 21S2-PCL films. These findings were confirmed by rapid increase in $\mathrm{pH}$ in the first month of incubation in PBS (Fig. 6a) associated with the high release rate of $\mathrm{Ca}^{2+}$ from A2-PCL films, what also correlate with the results obtained from the ICP-OES analysis of SBF (Fig. 3a). Our previous results, obtained during 14-month incubation of $21 \mathrm{~A} 2<45 \mu \mathrm{m}-\mathrm{PCL}$ and $21 \mathrm{~S} 2<45 \mu \mathrm{m}$-PCL composite films in UHQ water, showed that their mass loss practically did not depend on the chemical composition of glass modifiers [19]. As was expected, composites did not form a calcium phosphate layer upon immersion in UHQ water. These results would appear to support the conception that the kinetics of $\mathrm{CaP}$ layer deposition is one of the most important factors that affect the rate of composite degradation in PBS. This also suggests that the use of media for in vitro degradation tests, partially mimicking biological environment (e.g. content of inorganic species, $\mathrm{pH}$, buffering capacity), such as PBS, $\mathrm{SBF}$, allows us to explore additional aspects of degradation process and to better predict in vivo degradation behaviour of bioactive materials for tissue engineering. On the other hand, the changes in 
crystallinity of $21 \mathrm{~A} 2<45 \mu \mathrm{m}-\mathrm{PCL}$ and $21 \mathrm{~S} 2<45 \mu \mathrm{m}$ PCL composites incubated in UHQ water [19] showed the similar trend to those obtained for materials immersed in PBS. This, in turn, suggests that chemical composition of bioactive glass particles affected degradation of PCL matrix not only by changing the abilities of composites to form calcium phosphate layer on their surfaces.

When considering glass particle size, faster $\mathrm{CaP}$ layer formation in PBS occurred for composites containing particles of $<3 \mu \mathrm{m}$ size. Higher mass increase (Fig. 6b) persisting up to 3 months of incubation confirmed these observations. What is more, AS surface of $21 \mathrm{~S} 2<45 \mu \mathrm{m}$-PCL film showed no significant morphological changes. In the case of $21 \mathrm{~A} 2<3 \mu \mathrm{m}$-PCL material, the greater $\mathrm{pH}$ changes of PBS confirmed higher dissolution rate of $\mathrm{Ca}^{2+}$ from glass structure, favouring supersaturation of PBS and calcium phosphate layer formation (Fig. 6a). Furthermore, a much faster decrease in $\mathrm{pH}$ value confirmed faster deposition of CaP layer on the surfaces of this film, inhibiting further release of calcium ions to fresh PBS (solution was changed every month). This behaviour was a consequence of the uniform distribution of fillers in PCL matrix and thus their high exposure on both composite surfaces, in contrast to particles of $<45 \mu \mathrm{m}$ size, which sedimented near GS surfaces during film preparation process (Fig. 5). These findings are consistent with results obtained in our previous works $[25,26]$, as well as those of other authors [52, 53]. Moreover, it was shown that the larger surface area of smaller glass particles provides more effective ion release, enhancing the ability of HAp layer formation on the composite surfaces [22].

\section{Conclusions}

In present work, the short-term and long-term behaviour of poly( $\varepsilon$-caprolactone)/bioactive glass composites during incubation in biologically related fluids, namely SBF, DMEM-10\% FBS (up to 14 days) and PBS (up to 15 months), was investigated. Furthermore, normal human osteoblast response was also studied.

Obtained composite films showed excellent in vitro bioactivity, as evidenced by the formation of calcium phosphate layer on their surfaces upon immersion in SBF and DMEM-10\% FBS. However, kinetics of bioactivity process strongly depended on the type of the medium used. The layer of amino acids and proteins, derived from cell culture medium, on the surfaces of composites created barrier that inhibited release of the ions on the one hand, while increasing nucleation density of calcium phosphates, affecting the morphology of formed $\mathrm{CaP}$ layers on the other. The presence of bioactive glass fillers was shown to impart osteoinductive properties to obtained films, supporting osteoblast attachment and proliferation, as well as stimulating cell differentiation and also matrix mineralization process in vitro. We showed that kinetics of bioactivity process and also osteoinductive properties of composite films could be easily modulated with the use of different contents and chemical compositions of fillers.

The results showed that modification of PCL matrix with bioactive glass particles accelerated its degradation. We proved that the degradation rate of composites could be controlled and optimized for bone regeneration, in particular by using bioactive fillers causing different calcium phosphate layer forming ability on the surfaces of composites, depending on particle size and chemical composition.

We have presented new opportunities to design and obtain multifunctional composites with tunable degradation and bioactivity kinetics, as well as biological properties that can meet complex requirements of bone tissue engineering.

\section{Acknowledgements}

This work was supported by the National Science Centre, Poland, Grant No. 2015/17/N/ST8/00226 (MD). Michal Dziadek acknowledges financial support from the National Science Centre, Poland, under doctoral scholarship (2017/24/T/ST8/00041).

\section{Compliance with ethical standards}

Conflict of interest The authors declare that there is no conflict of interest.

Open Access This article is distributed under the terms of the Creative Commons Attribution 4.0 International License (http://creativecommons.org/ licenses/by/4.0/), which permits unrestricted use, distribution, and reproduction in any medium, provided you give appropriate credit to the original author(s) and the source, provide a link to the Creative Commons license, and indicate if changes were made. 


\section{References}

[1] Larrañaga A, Aldazabal P, Martin FJ, Sarasua JR (2014) Hydrolytic degradation and bioactivity of lactide and caprolactone based sponge-like scaffolds loaded with bioactive glass particles. Polym Degrad Stab 110:121-128. https://doi.org/10.1016/j.polymdegradstab.2014.08.021

[2] Lam CXF, Hutmacher DW, Schantz J-T et al (2009) Evaluation of polycaprolactone scaffold degradation for 6 months in vitro and in vivo. J Biomed Mater Res Part A 90A:906-919. https://doi.org/10.1002/jbm.a.32052

[3] Bottino MC, Thomas V, Schmidt G et al (2012) Recent advances in the development of GTR/GBR membranes for periodontal regeneration - a materials perspective. Dent Mater 28:703-721. https://doi.org/10.1016/j.dental.2012.04.022

[4] Hoornaert A, d'Arros C, Heymann M-F, Layrolle P (2016) Biocompatibility, resorption and biofunctionality of a new synthetic biodegradable membrane for guided bone regeneration. Biomed Mater 11:45012. https://doi.org/10.1088/ 1748-6041/11/4/045012

[5] Can E, Udenir G, Kanneci AI et al (2011) Investigation of PLLA/PCL blends and paclitaxel release profiles. AAPS PharmSciTech 12:1442-1453. https://doi.org/10.1208/ s12249-011-9714-y

[6] Chouzouri G, Xanthos M (2007) In vitro bioactivity and degradation of polycaprolactone composites containing silicate fillers. Acta Biomater 3:745-756. https://doi.org/10. 1016/j.actbio.2007.01.005

[7] Lei B, Shin K-H, Noh D-Y et al (2013) Sol-gel derived nanoscale bioactive glass (NBG) particles reinforced poly $(\varepsilon-$ caprolactone) composites for bone tissue engineering. Mater Sci Eng C 33:1102-1108. https://doi.org/10.1016/j.msec. 2012.11.039

[8] Lam CXF, Savalani MM, Teoh S-H, Hutmacher DW (2008) Dynamics of in vitro polymer degradation of polycaprolactone-based scaffolds: accelerated versus simulated physiological conditions. Biomed Mater 3:34108. https://doi.org/ 10.1088/1748-6041/3/3/034108

[9] Rich J, Jaakkola T, Tirri T et al (2002) In vitro evaluation of poly( $\varepsilon$-caprolactone-co-DL-lactide)/bioactive glass composites. Biomaterials 23:2143-2150. https://doi.org/10.1016/ S0142-9612(01)00345-3

[10] Díaz E, Sandonis I, Valle MB et al (2014) In vitro degradation of poly(caprolactone)/nHA composites. J Nanomater 2014:1-8. https://doi.org/10.1155/2014/802435

[11] Chouzouri G, Xanthos M (2007) Degradation of aliphatic polyesters in the presence of inorganic fillers. J Plast Film Sheeting 23:19-36. https://doi.org/10.1177/8756087907076599

[12] Łączka M, Cholewa-Kowalska K, Osyczka AM (2016) Bioactivity and osteoinductivity of glasses and glassceramics and their material determinants. Ceram Int 42:14313-14325. https://doi.org/10.1016/j.ceramint.2016.06.077

[13] Hench LL (2009) Genetic design of bioactive glass. J Eur Ceram Soc 29:1257-1265. https://doi.org/10.1016/j.jeurcer amsoc.2008.08.002

[14] Mami M, Lucas-Girot A, Oudadesse H et al (2008) Investigation of the surface reactivity of a sol-gel derived glass in the ternary system $\mathrm{SiO}_{2}-\mathrm{CaO}-\mathrm{P}_{2} \mathrm{O}_{5}$. Appl Surf Sci 254:7386-7393. https://doi.org/10.1016/j.apsusc.2008.05. 340

[15] Lee JTY, Leng Y, Chow KL et al (2011) Cell culture medium as an alternative to conventional simulated body fluid. Acta Biomater 7:2615-2622. https://doi.org/10.1016/j.actbio. 2011.02.034

[16] Bohner M, Lemaitre J (2009) Can bioactivity be tested in vitro with SBF solution? Biomaterials 30:2175-2179. https://doi.org/10.1016/j.biomaterials.2009.01.008

[17] Rohanová D, Boccaccini AR, Horkavcová D et al (2014) Is non-buffered DMEM solution a suitable medium for in vitro bioactivity tests? J Mater Chem B 2:5068-5076. https://doi. org/10.1039/C4TB00187G

[18] Łączka M, Cholewa K, Łạczka-Osyczka A (1997) Gelderived powders of $\mathrm{CaO}-\mathrm{P}_{2} \mathrm{O}_{5}-\mathrm{SiO}_{2}$ system as a starting material to production of bioactive ceramics. J Alloys Compd 248:42-51. https://doi.org/10.1016/S09258388(96)02648-5

[19] Dziadek M, Menaszek E, Zagrajczuk B et al (2015) New generation poly(e-caprolactone)/gel-derived bioactive glass composites for bone tissue engineering: part I. Material properties. Mater Sci Eng C 56:9-21. https://doi.org/10. 1016/j.msec.2015.06.020

[20] Kokubo T, Takadama H (2006) How useful is SBF in predicting in vivo bone bioactivity? Biomaterials 27:2907-2915. https://doi.org/10.1016/j.biomaterials.2006. 01.017

[21] Li W, Nooeaid P, Roether JA et al (2014) Preparation and characterization of vancomycin releasing PHBV coated 45S5 Bioglass ${ }^{\circledR}$-based glass-ceramic scaffolds for bone tissue engineering. J Eur Ceram Soc 34:505-514. https://doi. org/10.1016/j.jeurceramsoc.2013.08.032

[22] Caridade SG, Merino EG, Alves NM et al (2013) Chitosan membranes containing micro or nano-size bioactive glass particles: evolution of biomineralization followed by in situ dynamic mechanical analysis. J Mech Behav Biomed Mater 20:173-183. https://doi.org/10.1016/j.jmbbm.2012.11.012

[23] Poh PSP, Hutmacher DW, Stevens MM, Woodruff MA (2013) Fabrication and in vitro characterization of bioactive glass composite scaffolds for bone regeneration. Biofabrication 5:45005. https://doi.org/10.1088/1758-5082/5/4/ 045005 
[24] Misra SK, Ansari T, Mohn D et al (2010) Effect of nanoparticulate bioactive glass particles on bioactivity and cytocompatibility of poly(3-hydroxybutyrate) composites. J R Soc Interface 7:453-465. https://doi.org/10.1098/rsif. 2009.0255

[25] Dziadek M, Zagrajczuk B, Menaszek E et al (2017) Poly( $\varepsilon-$ caprolactone)-based membranes with tunable physicochemical, bioactive and osteoinductive properties. J Mater Sci 52:12960-12980. https://doi.org/10.1007/s10853-017-14248

[26] Dziadek M, Zagrajczuk B, Ziabka M et al (2016) The role of solvent type, size and chemical composition of bioactive glass particles in modulating material properties of poly $(\varepsilon-$ caprolactone) based composites. Compos Part A Appl Sci Manuf 90:90-99. https://doi.org/10.1016/j.compositesa. 2016.07.001

[27] Yoshioka T, Kamada F, Kawazoe N et al (2010) Structural changes and biodegradation of PLLA, PCL, and PLGA sponges during in vitro incubation. Polym Eng Sci 50:1895-1903. https://doi.org/10.1002/pen.21714

[28] Natu MV, de Sousa HC, Gil MH (2013) Influence of polymer processing technique on long term degradation of poly(e-caprolactone) constructs. Polym Degrad Stab 98:44-51. https://doi.org/10.1016/j.polymdegradstab.2012. 10.030

[29] Peña J, Corrales T, Izquierdo-Barba I, et al (2006) Long term degradation of poly( $\varepsilon$-caprolactone) films in biologically related fluids. https://doi.org/10.1016/j.polymdegradstab. 2005.10.016

[30] Bosworth LA, Downes S (2010) Physicochemical characterisation of degrading polycaprolactone scaffolds. Polym Degrad Stab 95:2269-2276. https://doi.org/10.1016/j.poly mdegradstab.2010.09.007

[31] Prabhakar RL, Brocchini S, Knowles JC (2005) Effect of glass composition on the degradation properties and ion release characteristics of phosphate glass-polycaprolactone composites. Biomaterials 26:2209-2218. https://doi.org/10. 1016/j.biomaterials.2004.07.016

[32] Kizuki T, Ohgaki M, Katsura M et al (2003) Effect of bonelike layer growth from culture medium on adherence of osteoblast-like cells. Biomaterials 24:941-947. https://doi. org/10.1016/S0142-9612(02)00430-1

[33] Lu HH, Pollack SR, Ducheyne P (2001) 45S5 Bioactive glass surface charge variations and the formation of a surface calcium phosphate layer in a solution containing fibronectin. J Biomed Mater Res 54:454-461. https://doi.org/10.1002/ 1097-4636(20010305)54:3<454:AID-JBM200>3.0.CO;2-H

[34] Juhasz JA, Best SM, Auffret AD, Bonfield W (2008) Biological control of apatite growth in simulated body fluid and human blood serum. J Mater Sci Mater Med 19:1823-1829. https://doi.org/10.1007/s10856-007-3344-7

[35] Wang K, Zhou C, Hong Y, Zhang X (2012) A review of protein adsorption on bioceramics. Interface Focus 2:259-277. https://doi.org/10.1098/rsfs.2012.0012

[36] Salinas AJ, Vallet-Regí M (2013) Bioactive ceramics: from bone grafts to tissue engineering. RSC Adv 3:11116-11131. https://doi.org/10.1039/c3ra00166k

[37] Iijima K, Sakai A, Komori A et al (2015) Control of biomimetic hydroxyapatite deposition on polymer substrates using different protein adsorption abilities. Colloids Surf B Biointerfaces 130:77-83. https://doi.org/10.1016/j.colsurfb. 2015.04.010

[38] Tavafoghi M, Brodusch N, Gauvin R, Cerruti M (2016) Hydroxyapatite formation on graphene oxide modified with amino acids: arginine versus glutamic acid. J R Soc Interface 13(114):20150986

[39] El-Kady AM, Saad EA, El-Hady BMA, Farag MM (2010) Synthesis of silicate glass/poly(l-lactide) composite scaffolds by freeze-extraction technique: characterization and in vitro bioactivity evaluation. Ceram Int 36:995-1009. https://doi. org/10.1016/j.ceramint.2009.11.012

[40] Fabbri P, Cannillo V, Sola A et al (2010) Highly porous polycaprolactone-45S5 Bioglass ${ }^{\circledR}$ scaffolds for bone tissue engineering. Compos Sci Technol 70:1869-1878. https://doi. org/10.1016/j.compscitech.2010.05.029

[41] Pamula E, Kokoszka J, Cholewa-Kowalska K et al (2011) Degradation, bioactivity, and osteogenic potential of composites made of PLGA and two different sol-gel bioactive glasses. Ann Biomed Eng 39:2114-2129. https://doi.org/10. 1007/s10439-011-0307-4

[42] Dziadek M, Pawlik J, Menaszek E et al (2015) Effect of the preparation methods on architecture, crystallinity, hydrolytic degradation, bioactivity, and biocompatibility of PCL/bioglass composite scaffolds. J Biomed Mater Res Part B Appl Biomater 103:1580-1593. https://doi.org/10.1002/jbm.b. 33350

[43] Zagrajczuk B, Dziadek M, Olejniczak Z et al (2017) Structural and chemical investigation of the gel-derived bioactive materials from the $\mathrm{SiO}_{2}-\mathrm{CaO}$ and $\mathrm{SiO}_{2}-\mathrm{CaO}-\mathrm{P}_{2} \mathrm{O}_{5}$ systems. Ceram Int 43:12742-12754. https://doi.org/10.1016/j.cer amint.2017.06.160

[44] Kim H-W, Lee H-H, Chun G-S (2008) Bioactivity and osteoblast responses of novel biomedical nanocomposites of bioactive glass nanofiber filled poly(lactic acid). J Biomed Mater Res Part A 85A:651-663. https://doi.org/10.1002/ jbm.a.31339

[45] Li W, Ding Y, Yu S et al (2015) Multifunctional chitosan45S5 bioactive glass-poly(3-hydroxybutyrate-co-3-hydroxyvalerate) microsphere composite membranes for guided 
tissue/bone regeneration. ACS Appl Mater Interfaces 7:20845-20854. https://doi.org/10.1021/acsami.5b06128

[46] Yao J, Radin S, Reilly G et al (2005) Solution mediated effect of bioactive glass in poly (lactic-co-glycolic acid)bioactive glass composites on osteogenesis of marrow stromal cells. Key Eng Mater 284-286:619-622. https://doi.org/ 10.4028/www.scientific.net/KEM.284-286.619

[47] Bosetti M, Zanardi L, Hench L, Cannas M (2003) Type I collagen production by osteoblast-like cells cultured in contact with different bioactive glasses. J Biomed Mater Res 64A:189-195. https://doi.org/10.1002/jbm.a.10415

[48] Silver IA, Deas J, Erecińska M (2001) Interactions of bioactive glasses with osteoblasts in vitro: effects of 45S5 Bioglass $^{\circledR}$, and $58 \mathrm{~S}$ and $77 \mathrm{~S}$ bioactive glasses on metabolism, intracellular ion concentrations and cell viability. Biomaterials 22:175-185. https://doi.org/10.1016/S01429612(00)00173-3

[49] Gentleman MM, Gentleman E (2014) The role of surface free energy in osteoblast-biomaterial interactions. Int Mater Rev 59:417-429. https://doi.org/10.1179/1743280414Y. 0000000038
[50] Maquet V, Boccaccini AR, Pravata L et al (2004) Porous poly $\left(\alpha\right.$-hydroxyacid)/Bioglass ${ }^{\circledR}$ composite scaffolds for bone tissue engineering. I: preparation and in vitro characterisation. Biomaterials 25:4185-4194. https://doi.org/10.1016/j. biomaterials.2003.10.082

[51] Mohammadi MS, Ahmed I, Muja N et al (2012) Effect of Si and Fe doping on calcium phosphate glass fibre reinforced polycaprolactone bone analogous composites. Acta Biomater 8:1616-1626. https://doi.org/10.1016/j.actbio.2011. 12.030

[52] Tamjid E, Bagheri R, Vossoughi M, Simchi A (2011) Effect of particle size on the in vitro bioactivity, hydrophilicity and mechanical properties of bioactive glass-reinforced polycaprolactone composites. Mater Sci Eng C 31:1526-1533. https://doi.org/10.1016/j.msec.2011.06.013

[53] Jaakkola T, Rich J, Tirri T et al (2004) In vitro Ca-P precipitation on biodegradable thermoplastic composite of poly( $\varepsilon$-caprolactone-co-dl-lactide) and bioactive glass (S53P4). Biomaterials 25:575-581. https://doi.org/10.1016/ S0142-9612(03)00558-1 